\title{
Positive solutions for $P$-Laplace problems with nonlinear time-fractional differential equation
}

\author{
Meilan Qiu', Liquan Mei $^{1 *}$, Ganshan Yang ${ }^{2,3^{*}}$ and George Xianzhi Yuan ${ }^{4 *}$
}

Dedicated to the third and fourth authors' supervisor, Professor Shisheng Zhang, for his 80th birthday

*Correspondence:

lqmei@mail.xjtu.edu.cn;

ganshanyang@aliyun.com;

george_yuan99@tongji.edu.cn

${ }^{1}$ School of Mathematics and

Statistics, Xi'an Jiaotong University,

Xi'an, 710049, China

${ }^{2}$ Department of Mathematics,

Yunnan Nationalities University,

Kunming, 650031, China

Full list of author information is

available at the end of the article

\begin{abstract}
In this paper, we study the existence and multiplicity of positive solutions for semi-linear elliptic equations with a sign-changing weight function in weighted Sobolev spaces. By investigating the compact embedding theorem and based on the extraction of the Palais-Smale sequence in the Nehari manifold which is a subset of the weighted Sobolev spaces, we derive the existence of the multiple positive solutions of the equations by using the variational method. In the last part of this paper, by applying the Arzela-Ascoli fixed point theorem, some existence results of the corresponding time-fractional equations for semi-linear elliptic equations are obtained.
\end{abstract}

MSC: 35Q30; 76D05; 76D10

Keywords: Nehari manifold; concave-convex nonlinearities; positive weight function; weighted Sobolev space; time-fractional equation; fixed point theorem

\section{Introduction}

In this paper, we consider the multiplicity results of positive solutions for the following semi-linear problem:

$$
\left(E_{\lambda f, h}\right) \begin{cases}-\operatorname{div}\left(a(x)|\nabla u(x)|^{p-2} \nabla u(x)\right)+(u(x))^{p-1} & \\ =\lambda f(x)|u(x)|^{q-1}+h(x)|u(x)|^{r-1}, & \text { in } \Omega, \\ u(x)=0, & \text { on } \partial \Omega\end{cases}
$$

$u \in W_{0}^{1, p}(a, \Omega)$, where $\lambda$ is a real positive parameter, $1<q<p<r<p_{s}^{*}\left(p>2, p_{s}=\frac{p s}{s+1}, p_{s}^{*}=\right.$ $\left.\frac{N p_{s}}{N-p_{s}}, s \in\left(\frac{N}{p}, \infty\right) \cap\left[\frac{1}{p-1}, \infty\right), p s<N(s+1)\right) . \Omega$ is a bounded region with smooth boundary in $\mathbf{R}^{N} ; a(x), f(x), h(x)$ are measurable functions and satisfy the following conditions:

$\left(\mathrm{H}_{1}\right) \quad 0 \leq f(x) \in L^{H}(\Omega)$, where $L^{H}(\Omega)=L^{\frac{r}{r-q}}(\Omega), q<r<p_{s}^{*}$, and $f(x)$ has a compact support in $\Omega$,

$\left(\mathrm{H}_{2}\right) \quad 0 \leq h(x) \in L^{\infty}(\Omega)$, and it satisfies $h(x) \rightarrow 1$ as $|x| \rightarrow \infty$,

$\left(\mathrm{H}_{3}\right) a(x)$ is a positive weight function, locally Hölder continuous, and almost everywhere with positive measure in the Sobolev space $W_{0}^{1, p}(a(x), \Omega)$ which comes with the standard norm $\|u\|=\left\{\int_{\Omega}\left(a|\nabla u(x)|^{p}+(u(x))^{p}\right) d x\right\}^{\frac{1}{p}}$ and there exists $v(x)$ if and only if 


$$
\begin{aligned}
& \frac{v(x)}{c_{1}} \leq a(x) \leq c_{1} v(x) \text {, where } c_{1} \geq 1 \text { and } v(x) \text { is another weight function, which satisfies } \\
& v(x) \in L_{\mathrm{loc}}^{1}(\Omega), v(x)^{-\frac{1}{p-1}} \in L_{\mathrm{loc}}^{1}(\Omega), v(x)^{-s} \in L^{1}(\Omega) .
\end{aligned}
$$

The problem $\left(E_{\lambda f, h}\right)$ is an important and basic mathematical model, widely used in many fields. For specific theoretical implications of the above model, one can refer to Drábek, Kufner and Nicolosi [1] and Adams and John [2] and references wherein.

Analogous equations with nonlinearities concave-convex in bounded domains are widely studied. For example, Ambrosetti [3] studied the problem below:

$$
\left(E_{\lambda}\right) \begin{cases}-\Delta u=\lambda|u|^{q}+|u|^{p}, & \text { in } \Omega, \\ u>0, & \text { in } \Omega, \\ u=0, & \text { on } \partial \Omega,\end{cases}
$$

where $0<q<1<p \leq 2^{*}-1$. They proved the existence of $\lambda_{0}>0$ such that the problem $\left(E_{\lambda}\right)$ admits at least two positive solutions for $\lambda \in\left(0, \lambda_{0}\right)$; there is one positive solution for $\lambda=\lambda_{0}$, and no positive solution exists for $\lambda>\lambda_{0}$. Recently, for $\Omega=B_{N}(0,1)$, that is, $\Omega$ is a unit ball, Adimurthi and Yadava [4], Damascelli et al. [5] and Tang [6] proved that there are exactly two solutions for $\lambda \in\left(0, \lambda_{0}\right)$; one positive solution for $\lambda=\lambda_{0}$ and no positive solution exists for $\lambda>\lambda_{0}$. When $p \equiv 2, h(x)=1$ and $a(x) \equiv 1$, Wu [7] has investigated equation $\left(E_{\lambda f, 1}\right)$, and he found that there exists $\lambda_{0}>0$ such that equation $\left(E_{\lambda f, 1}\right)$ admits at least two positive solutions for $\lambda \in\left(0, \lambda_{0}\right)$. Among other interesting results, Miotto and Miyagaki [8] have studied the following equation:

$$
\begin{cases}-\Delta u+u=\lambda f(x)|u|^{q-1}+h(x)|u|^{p-1}, & \text { in } \Omega, \\ u \equiv 0, & \text { on } \partial \Omega\end{cases}
$$

where $\lambda>0,1<q<2<p<2^{*}\left(2^{*}=\frac{2 N}{N-2}\right.$ if $N \geq 3,2^{*}=\infty$ if $\left.N=2\right), \Omega=\Omega^{\prime} \times R\left(\Omega^{\prime} \subset\right.$ $\left.R^{N-1}\right)$ is an infinite strip domains, assuming that $f(x) \in L^{\frac{r}{r-q}}(\Omega)=L^{1}$, where $q<r \leq 2^{*}$, with $f^{+} \not \equiv 0$ and $f^{-}$is bounded and has a compact support in $\Omega .0 \leq h(x) \in L^{\infty}(\Omega)$ satisfies $\lim _{\left|x_{N}\right| \rightarrow \infty} h\left(x^{\prime}, x_{N}\right)=1$ and there exists $c_{0}>0, \theta_{1}$ being the first eigenvalue of the Dirichlet problem $-\Delta$ in $\Omega^{\prime}$, such that

$$
h\left(x^{\prime}, x_{N}\right) \geq 1-c_{0} e^{-2 \sqrt{1+\theta_{1}}\left|x_{N}\right|}
$$

for all $\mathbf{x}=\left(x^{\prime}, x_{N}\right) \in \Omega$. They proved that the existence of $\Lambda=\Lambda\left(q, p,\|h\|_{L^{\infty}}, r\right)$ such that the problem (1.1) has at least two positive solutions for all $\lambda \in\left(0, \Lambda\|f\|_{L^{1}}^{-1}\right)$, Wu in [9] has studied (1.1) under the assumption that $0 \supsetneqq f \in L^{\frac{2}{2-q}}(\Omega), 0<h \in C(\Omega)$ satisfying $\lim _{\left|x_{N}\right| \rightarrow \infty} h(\mathbf{x})=1$ in $\Omega=\Omega^{\prime} \times R$ and there exist $\delta>0$ and $0<c_{0}<1$ such that

$$
h\left(x^{\prime}, x_{N}\right) \geq 1-c_{0} e^{-2 \sqrt{1+\theta_{1}+\delta}\left|x_{N}\right|},
$$

for all $\left(x^{\prime}, x_{N}\right) \in \Omega$. The existence of $\Lambda_{0}>0$ was obtained such that for $\lambda \in\left(0, \Lambda_{0}\right)$ the problem (1.1) possesses at least two positive solutions.

We consider the $P$-Laplace Dirichlet problem above. In the following we will switch our view point to investigate the existence of positive solutions for the corresponding nonlinear time-fractional differential equation of the problem $\left(E_{\lambda f, h}\right)$. We know that the subject 
of fractional differential equations has emerged as an important area of investigation by the fact that it has numerous applications in viscoelasticity, electrochemistry, control, electromagnetic, porous media, and so on, thus the subject of fractional differential equations is gaining much importance and attention. For some recent developments on the subject, please see the literature [10-17], and the references therein for more details. But not many people pay attention to the study of the $P$-Laplace problems with nonlinear partial differential equations of time-fractional order, except the literature such as $[18,19]$, but the aim of this paper is to do so, too. To the best of our knowledge, the results in this paper are new and original as we have not found any discussion in the existing literature.

The paper is organized as follows. In Sections 2 and 3, we show that equation $\left(E_{\lambda f, h}\right)$ in weighted Sobolev space has at least two positive solutions for $\lambda$ sufficiently small. First, we recall the Nehari manifold which is a subset of the weighted Sobolev space, and analyze the behavior of the energy functional associated with our problems on the Nehari manifold. Moreover, by extracting the Palais-Smale sequences in the Nehari manifold and combining the properties of the compact embedding theorem in weighted Sobolev space, we obtain the result that there exist at least two positive solutions of the problem $\left(E_{\lambda f, h}\right)$. In Section 4, we shall consider the following time-fractional differential equations derived from $\left(E_{\lambda f, h}\right)$ :

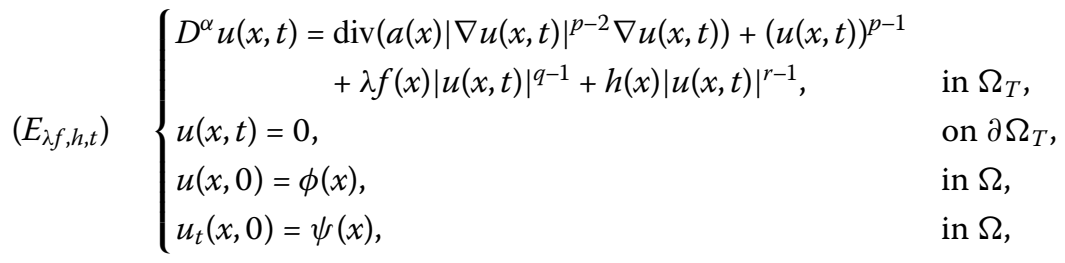

where $\Omega_{T}=\Omega \times[0, T], D^{\alpha}$ denotes the Caputo fractional derivative (e.g., see [13]), $1<\alpha<2$ is a parameter describing the order of the fractional time, and $\phi(x), \psi(x) \in H_{0}^{1}(a(x), \Omega)$ are given real-valued functions. Then the problem $\left(E_{\lambda f, h, t}\right)$ is deduced to an equivalent integral equation under the fractional order integral operator $I^{\alpha}$. Finally, we prove the existence of solution for the time-fractional differential equations by using the Arzela-Ascoli fixed point theorem. The conclusion is given by Section 5 .

\section{Notations and preliminaries}

In the following, we first consider the positive solutions of the following problem.

Theorem 2.1 There exists $\lambda_{0}=\lambda_{0}\left(q, p,\|h\|_{\infty}, r,\|f\|_{L^{H}}^{-1}\right)>0$ such that for $\lambda \in\left(0, \lambda_{0}\right)$, the equation $\left(E_{\lambda f, h}\right)$ has at least two positive solutions.

In order to prove it, we need the following lemma.

Lemma 2.2 If $2<p<r<p_{s}^{*}, X \hookrightarrow L^{r}(\Omega)$ is the compact embedding, then $X \hookrightarrow L^{p}(\Omega)$ is also the compact embedding, where $X=W_{0}^{1, p}(a(x), \Omega)$ (e.g., see Drábek, Kufner and Nicolosi [1]).

Throughout this section, we denote by $S_{r}$ the best Sobolev constant for the embedding of $W_{0}^{1, p}(a(x), \Omega)$ in $L^{r}(\Omega)$. We define

$$
S_{r}=\sup _{u \in W_{0}^{1, p}(a(x), \Omega) \backslash\{0\}}\left\{\frac{\|u\|_{L^{r}}}{\|u\|_{X}}\right\} .
$$


For the convenience we will denote $W_{0}^{1, p}(a(x), \Omega)$ by $X$, and $\|\cdot\|_{X}$ by $\|\cdot\|$ if there is no confusion, unless otherwise stated, and the integrals are over $\Omega$. Now we give the proof of Theorem 2.1.

Proof Associated with equation $\left(E_{\lambda f, h}\right)$, we define the energy functional $I_{\lambda}$ in $X$ for given $\lambda \geq 0, f(x)$ and $h(x)$ by

$$
I_{\lambda}(u)=\frac{1}{p} \int_{\Omega}\left(a|\nabla u|^{p}+u^{p}\right) d x-\frac{1}{q} \lambda \int_{\Omega}\left(f(x)|u|^{q}\right) d x-\frac{1}{r} \int_{\Omega}\left(h(x)|u|^{r}\right) d x .
$$

It is clear that $I_{\lambda}$ is of class $C^{1}$ with Gâteaux derivative $I_{\lambda}^{\prime}(u)$ at each $u \in X$ given by

$$
\begin{aligned}
\left\langle I_{\lambda}^{\prime}(u), \varphi\right\rangle= & \int_{\Omega}\left(a|\nabla u|^{p-1} \nabla \varphi+u^{p-1} \varphi\right) d x-\lambda \int_{\Omega}\left(f|u|^{q-2} u \varphi\right) d x \\
& -\int_{\Omega}\left(h|u|^{r-2} u \varphi\right) d x,
\end{aligned}
$$

for all $\varphi \in X$. Therefore the (weak) solutions of equation $\left(E_{\lambda f, h}\right)$ are the critical points of the energy functional $I_{\lambda}$ (see Rabinowitz [20]).

As the energy functional $I_{\lambda}$ is not bounded from below on $X$, it is useful to consider the functional on the Nehari manifold which has the best behavior subset of $X$ (see Brown and Zhang [21]). For any $\lambda>0$, we define

$$
N_{\lambda}=\left\{u \in X \backslash\{0\}:\left\langle I_{\lambda}^{\prime}(u), u\right\rangle=0\right\} .
$$

Then $u \in N_{\lambda}$ if and only if

$$
\left\langle I_{\lambda}^{\prime}(u), u\right\rangle=\|u\|^{p}-\lambda \int_{\Omega}\left(f|u|^{q}\right) d x-\int_{\Omega}\left(h|u|^{r}\right) d x=0 .
$$

Note that any nonzero solution of the problem $\left(E_{\lambda f, h}\right)$ belongs to $N_{\lambda}$. Furthermore, we have the following result.

Lemma 2.3 The functional $I_{\lambda}$ is coercive and bounded from below on $N_{\lambda}$.

Proof Let $u \in N_{\lambda}$ be arbitrary. Then by (2.1) and by the Hölder and Sobolev inequalities we get

$$
\begin{aligned}
I_{\lambda}(u) & \geq \frac{r-p}{p r}\|u\|^{p}-\left(\frac{r-q}{q r}\right) \lambda\left(\int_{\Omega}|f|^{\frac{r}{r-q}} d x\right)^{\frac{r-q}{r}}\left(\int_{\Omega}|u|^{r} d x\right)^{\frac{q}{r}} \\
& \geq \frac{r-p}{p r}\|u\|^{p}-\left(\frac{r-q}{q r}\right) \lambda\|f\|_{L^{H}} S_{r}^{q}\|u\|^{q} .
\end{aligned}
$$

Since $q<p<r$, it follows that $I_{\lambda}$ is bounded from below and coercive on $N_{\lambda}$ provided $\lambda$ is small enough. 
Next, we consider the Nehari minimization problem; for $\lambda \geq 0$, define $\alpha_{\lambda}=\inf _{u \in N_{\lambda}} I_{\lambda}(u)$ and

$$
\psi_{\lambda}(u)=\left\langle I_{\lambda}^{\prime}(u), u\right\rangle=\|u\|^{p}-\lambda \int_{\Omega}\left(f|u|^{q}\right) d x-\int_{\Omega}\left(h|u|^{r}\right) d x .
$$

Then for $u \in N_{\lambda}$ by (2.1) we have

$$
\begin{aligned}
\left\langle\psi_{\lambda}^{\prime}(u), u\right\rangle & =p\|u\|^{p}-q \lambda \int_{\Omega}\left(f|u|^{q}\right) d x-r \int_{\Omega}\left(h|u|^{r}\right) d x \\
& =(p-q)\|u\|^{p}-(r-q) \int_{\Omega}\left(h|u|^{r}\right) d x \\
& =(p-r)\|u\|^{p}-(q-r) \lambda \int_{\Omega}\left(f|u|^{q}\right) d x .
\end{aligned}
$$

Now, we split $N_{\lambda}$ into three parts (see Drábek, Kufner and Nicolosi [1] and Ambrosetti et al. [3]).

$$
\begin{aligned}
& N_{\lambda}^{+}=\left\{u \in N_{\lambda}:\left\langle\psi_{\lambda}^{\prime}(u), u\right\rangle>0\right\}, \\
& N_{\lambda}^{0}=\left\{u \in N_{\lambda}:\left\langle\psi_{\lambda}^{\prime}(u), u\right\rangle=0\right\}, \\
& N_{\lambda}^{-}=\left\{u \in N_{\lambda}:\left\langle\psi_{\lambda}^{\prime}(u), u\right\rangle<0\right\} .
\end{aligned}
$$

Then we have the following result.

Lemma 2.4 There exists $\lambda_{1}>0$ such that for each $\lambda \in\left(0, \lambda_{1}\right)$, we have $N_{\lambda}^{0}=\emptyset$.

Proof We consider the following two cases.

Case (I) $u \in N_{\lambda}(\Omega)$ and $\int_{\Omega}\left(f(x)|u|^{q}\right) d x=0$. We then have

$$
\|u\|^{p}-\int_{\Omega}\left(h|u|^{r}\right) d x=0
$$

Thus

$$
\begin{aligned}
\left\langle\psi_{\lambda}^{\prime}(u), u\right\rangle & =(p-q)\|u\|^{p}-(r-q) \int_{\Omega}\left(h|u|^{r}\right) d x \\
& =[(p-q)-(r-q)]\|u\|^{p}=(p-r)\|u\|^{p}<0 .
\end{aligned}
$$

Hence $u \notin N_{\lambda}^{0}(\Omega)$.

Case (II) $u \in N_{\lambda}(\Omega)$ and $\int_{\Omega}\left(f(x)|u|^{q}\right) d x \neq 0$. Suppose that $N_{\lambda}^{0} \neq \emptyset$ for all $\lambda>0$. If $u \in N_{\lambda}^{0}$, then we have

$$
\begin{aligned}
0 & =\left\langle\psi_{\lambda}^{\prime}(u), u\right\rangle=p\|u\|^{p}-q \lambda \int_{\Omega}\left(f|u|^{q}\right) d x-r \int_{\Omega}\left(h|u|^{r}\right) d x \\
& =(p-q)\|u\|^{p}-(r-q) \int_{\Omega}\left(h|u|^{r}\right) d x .
\end{aligned}
$$

Thus

$$
0<\|u\|^{p}=\frac{r-q}{p-q} \int_{\Omega}\left(h|u|^{r}\right) d x
$$


and

$$
0<\lambda \int_{\Omega}\left(f|u|^{q}\right) d x=\|u\|^{p}-\int_{\Omega}\left(h|u|^{r}\right) d x=\frac{r-p}{p-q} \int_{\Omega}\left(h|u|^{r}\right) d x .
$$

Moreover, by the Hölder and Sobolev inequalities, for all $u \in X$, we obtain

$$
\lambda \int_{\Omega}\left(f|u|^{q}\right) d x \leq \lambda\|f\|_{L^{H}} S_{r}^{q}\|u\|^{q} .
$$

Thus for any $u \in N_{\lambda}^{0}$, by (2.4)-(2.6) we obtain

$$
\|u\| \leq\left(\frac{r-q}{r-p}\right)^{\frac{1}{p-q}} S_{r}^{\frac{q}{p-q}}\left(\lambda\|f\|_{L^{H}}\right)^{\frac{1}{p-q}} .
$$

Let $J_{\lambda}: N_{\lambda}(\Omega) \rightarrow \mathbf{R}$ be given by

$$
J_{\lambda}(u)=K(q, r)\left(\frac{\|u\|^{r}}{\int_{\Omega}\left(h|u|^{r}\right) d x}\right)^{\frac{p}{r-p}}-\lambda \int_{\Omega}\left(f|u|^{q}\right) d x,
$$

where $K(q, r)=\left(\frac{r-p}{p-q}\right)\left(\frac{p-q}{r-q}\right)^{\frac{r}{r-p}}$. Then $J_{\lambda}(u)=0$ for all $u \in N_{\lambda}^{0}$. Indeed, from (2.4)-(2.5), it follows that, for $u \in N_{\lambda}^{0}$, we have

$$
\begin{aligned}
J_{\lambda}(u) & =K(q, r)\left(\frac{\|u\|^{r}}{\int_{\Omega}\left(h|u|^{r}\right) d x}\right)^{\frac{p}{r-p}}-\lambda \int_{\Omega}\left(f|u|^{q}\right) d x \\
& =\left(\frac{r-p}{p-q}\right)\left(\frac{p-q}{r-q}\right)^{\frac{r}{r-p}} \frac{\left(\|u\|^{p}\right)^{\frac{r}{r-p}}}{\left(\int_{\Omega}\left(h|u|^{r}\right) d x\right)^{\frac{p}{r-p}}}-\frac{r-p}{p-q} \int_{\Omega}\left(h|u|^{r}\right) d x \\
& =\left(\frac{r-p}{p-q}\right)\left(\frac{p-q}{r-q}\right)^{\frac{r}{r-p}} \frac{\left(\frac{r-q}{p-q}\right)^{\frac{r}{r-p}}\left(\int_{\Omega}\left(h|u|^{r}\right) d x\right)^{\frac{r}{r-p}}}{\left(\int_{\Omega}\left(h|u|^{r}\right) d x\right)^{\frac{p}{r-p}}}-\frac{r-p}{p-q} \int_{\Omega}\left(h|u|^{r}\right) d x \\
& =\frac{r-p}{p-q} \int_{\Omega}\left(h|u|^{r}\right) d x-\frac{r-p}{p-q} \int_{\Omega}\left(h|u|^{r}\right) d x=0 .
\end{aligned}
$$

However, by (2.7) and the Hölder and Sobolev inequalities, for $u \in N_{\lambda}^{0}$,

$$
\begin{aligned}
J_{\lambda}(u) & \geq K(q, r)\left(\frac{\|u\|^{r}}{\int_{\Omega}\left(h|u|^{r}\right) d x}\right)^{\frac{p}{r-p}}-\lambda\|f\|_{L^{H}} S_{r}^{r}\|u\|^{r} \\
& \geq K(q, r)(\tilde{c})^{\frac{p}{r-p}}-\lambda\|f\|_{L^{H}} S_{r}^{r}\|u\|^{r},
\end{aligned}
$$

where $\tilde{c}=\left(\|h\|_{L^{\infty}} S_{r}^{r}\right)^{-1}$. This implies that for $\lambda$ sufficiently small we have $J_{\lambda}(u)>0$ for all $u \in N_{\lambda}^{0}$, this contradicts (2.8). Thus, we can conclude that there exists $\lambda_{1}>0$ such that for $\lambda \in\left(0, \lambda_{1}\right)$, we have $N_{\lambda}^{0}=\emptyset$. The proof is complete.

Lemma 2.5 If $u \in N_{\lambda}^{+}$, then $\int_{\Omega}\left(f|u|^{q}\right) d x>0$.

Proof We have

$$
\|u\|^{p}-\lambda \int_{\Omega}\left(f|u|^{q}\right) d x-\int_{\Omega}\left(h|u|^{r}\right) d x=0
$$


and

$$
\|u\|^{p}>\frac{r-q}{p-q} \int_{\Omega}\left(h|u|^{r}\right) d x .
$$

Thus

$$
\lambda \int_{\Omega}\left(f|u|^{q}\right) d x=\|u\|^{p}-\int_{\Omega}\left(h|u|^{r}\right) d x>\frac{r-p}{p-q} \int_{\Omega}\left(h|u|^{r}\right) d x>0,
$$

and this completes the proof.

By Lemma 2.4, for $\lambda \in\left(0, \lambda_{1}\right)$ we write

$$
N_{\lambda}=N_{\lambda}^{+} \cup N_{\lambda}^{-}
$$

and we define

$$
\begin{aligned}
& \alpha_{\lambda}^{+}(\Omega)=\inf _{u \in N_{\lambda}^{+}} I_{\lambda}(u), \\
& \alpha_{\lambda}^{-}(\Omega)=\inf _{u \in N_{\lambda}^{-}} I_{\lambda}(u) .
\end{aligned}
$$

The following results show that minimizers on $N_{\lambda}$ are the 'usual' critical points for $I_{\lambda}$.

Lemma 2.6 For $\lambda \in\left(0, \lambda_{1}\right)$, if $u_{0} \in N_{\lambda}$ is a local minimizer point for $I_{\lambda}$ on $N_{\lambda}$ and $u_{0} \notin N_{\lambda}^{0}$, then $I_{\lambda}^{\prime}\left(u_{0}\right)=0$ in $X^{-1}(\Omega)$.

Proof If $u_{0}$ is a local minimizer point for $I_{\lambda}$ on $N_{\lambda}$, then $u_{0}$ is a solution of the following optimization problem:

$$
\inf _{\psi_{\lambda}(u)=0} I_{\lambda}(u)
$$

Hence, by the theory of Lagrange multipliers, there exists $\theta \in \mathbf{R}$ such that

$$
I_{\lambda}^{\prime}\left(u_{0}\right)=\theta \psi_{\lambda}^{\prime}\left(u_{0}\right)
$$

in $X^{-1}(\Omega)$. Thus

$$
\left\langle I_{\lambda}^{\prime}\left(u_{0}\right), u_{0}\right\rangle=\theta\left\langle\psi_{\lambda}^{\prime}\left(u_{0}\right), u_{0}\right\rangle
$$

Since $u_{0} \in N_{\lambda},\left\langle I_{\lambda}^{\prime}\left(u_{0}\right), u_{0}\right\rangle=0$ and so

$$
\left\|u_{0}\right\|^{p}-\lambda \int_{\Omega}\left(f\left|u_{0}\right|^{q}\right) d x-\int_{\Omega}\left(h\left|u_{0}\right|^{r}\right) d x=0
$$

Hence

$$
\left\langle\psi_{\lambda}^{\prime}\left(u_{0}\right), u_{0}\right\rangle=(p-q)\left\|u_{0}\right\|^{p}-(r-q) \int_{\Omega}\left(h\left|u_{0}\right|^{r}\right) d x
$$

Thus, if $u_{0} \notin N_{\lambda}^{0},\left\langle\psi_{\lambda}^{\prime}\left(u_{0}\right), u_{0}\right\rangle \neq 0$ and so by (2.9), $\theta=0$. This completes the proof. 
For each $u \in N_{\lambda} \subset X \backslash\{0\}$, we have

$$
\|u\|^{p}-\lambda \int_{\Omega}\left(f|u|^{q}\right) d x-\int_{\Omega}\left(h|u|^{r}\right) d x=0
$$

so we have $\lambda \int_{\Omega}\left(f|u|^{q}\right) d x=\|u\|^{p}-\int_{\Omega}\left(h|u|^{r}\right) d x$. By (2.2), we define the fiber map $\phi_{u}(t)=$ $I_{\lambda}(t u)$, and we let $\phi_{u}^{\prime}(t)=0$, i.e.,

$$
t^{p-1}\|u\|^{p}-t^{q-1} \lambda \int_{\Omega}\left(f|u|^{q}\right) d x-t^{r-1} \int_{\Omega}\left(h|u|^{r}\right) d x=0 .
$$

Hence

$$
\left(t^{p-q}-1\right)\|u\|^{p}-\left(t^{r-q}-1\right) \int_{\Omega}\left(h|u|^{r}\right) d x=0 .
$$

By the Lagrange mean theorem, there exists a $t(\xi)$ such that

$$
\frac{\|u\|^{p}}{\int_{\Omega}\left(h|u|^{r}\right) d x}=\frac{t^{r-q}-1}{t^{p-q}-1}=\frac{(r-q) t(\xi)^{r-q-1}}{(p-q) t(\xi)^{p-q-1}}=\frac{r-q}{p-q} t(\xi)^{r-p} .
$$

In particular, we have

$$
0<t_{\max }(u)=t(\xi)=\left(\frac{(p-q)\|u\|^{p}}{(r-q) \int_{\Omega}\left(h|u|^{r}\right) d x}\right)^{\frac{1}{r-p}} .
$$

Lemma 2.7 Let $H=\frac{r}{r-q}$ and $\lambda_{2}=\left(\frac{r}{r-q}\right)\left(\frac{p-q}{r-q}\right)^{\frac{p-q}{r-p}} S^{\frac{2(q-r)}{r-p}}\left(\|f\|_{L^{H}}\right)^{-1}$. Then for each $u \in X \backslash\{0\}$ and $\lambda \in\left(0, \lambda_{2}\right)$, we have

(i) there is a unique $t^{-}=t^{-}(u)>t_{\max }>0$ such that $t^{-}(u) u \in N_{\lambda}^{-}$and

$$
I_{\lambda}\left(t^{-} u\right)=\sup _{t \geq t_{\max }} I_{\lambda}(t u)>0
$$

(ii) if $\int_{\Omega}\left(f(x)|u|^{q}\right) d x>0$, then there exists unique $0<t^{+}=t^{+}(u)<t_{\max }$ such that $t^{+}(u) u \in N_{\lambda}^{+}$and

$$
I_{\lambda}\left(t^{+} u\right)=\inf _{0 \leq t \leq t_{\max }} I_{\lambda}(t u)
$$

(iii) there exists a continuous bijection between $U=\{u \in X \backslash\{0\}:\|u\|=1\}$ and $N_{\lambda}^{-}$, in particular, $t^{-}(u)$ is a continuous function for nonzero $u$.

Proof (i) Fix $u \in X \backslash\{0\}$, let

$$
s(t)=t^{p-q}\|u\|^{p}-t^{r-q} \int_{\Omega}\left(h(x)|u|^{r}\right) d x,
$$

for $t \geq 0$, we have $s(0)=0, s(t) \rightarrow(-\infty)$ as $t \rightarrow \infty$, and by (2.2), we have

$$
s(t)=t^{p-q}\|u\|^{p}-t^{r-q}\left(\frac{p-q}{r-q}\right)\|u\|^{p}=\left(t^{p-q}-t^{r-q} \frac{p-q}{r-q}\right)\|u\|^{p},
$$


since $1<q<p<r$, so $0<\frac{p-q}{r-q}<1, p-q<r-q$, hence, if $0<t<1$, then $t^{p-q}>t^{r-q}$, and so $s(t)<0$, therefore, $s(t)$ can achieve its maximum at $t_{\max }$. Moreover,

$$
\begin{aligned}
s\left(t_{\max }\right) & \geq t^{p-q}\|u\|^{p}-t^{r-q}\|h\|_{L^{\infty}} S_{r}^{r}\|u\|^{r} \\
& \geq\left(\frac{p-q}{r-q}\right)^{\frac{p-q}{r-p}} \frac{\|u\|^{\frac{p(r-q)}{r-p}}}{\left(\tilde{c}^{-1}\|u\|^{r}\right)^{\frac{p-q}{r-p}}}-\left(\tilde{c}^{-1}\right)\left(\frac{p-q}{r-q}\right)^{\frac{r-q}{r-p}} \frac{\|u\|^{\frac{r^{2}-p q}{r-p}}}{\left(\tilde{c}^{-1}\right)^{\frac{r-q}{r-p}}\|u\|^{\frac{r^{2}-q r}{r-p}}} \\
& \geq\left(\frac{p-q}{r-q}\right)^{\frac{p-q}{r-p}}(\tilde{c})^{\frac{p-q}{r-p}}\|u\|^{q}-\left(\tilde{c}^{-1}\right)^{\frac{q-p}{r-p}}\left(\frac{p-q}{r-q}\right)^{\frac{r-q}{r-p}}\|u\|^{q} \\
& \geq K(q, r)(\tilde{c})^{\frac{p-q}{r-p}}\|u\|^{q} \\
& \geq S_{r}^{q} \lambda\|f\|_{L^{H}}\|u\|^{q} \\
& \geq \lambda \int_{\Omega}\left(f|u|^{q}\right) d x .
\end{aligned}
$$

Note that $t u \in N_{\lambda}$, if and only if $s(t)=\lambda \int_{\Omega}\left(f|u|^{q}\right) d x$.

Case (I) Suppose that $\lambda \int_{\Omega}\left(f|u|^{q}\right) d x \leq 0$, there is a unique $t^{-}>t_{\max }$ such that $s\left(t^{-}\right)=$ $\lambda \int_{\Omega}\left(f|u|^{q}\right) d x$ and $s^{\prime}\left(t^{-}\right)<0$. Now

$$
\begin{aligned}
& (p-q)\left\|t^{-} u\right\|^{p}-(r-q) \int_{\Omega}\left(h\left|t^{-} u\right|^{r}\right) d x \\
& \quad=\left(t^{-}\right)^{q+1}\left[(p-q)\left(t^{-}\right)^{p-q-1}\|u\|^{p}-(r-q) t^{r-q-1} \int_{\Omega}\left(h\left|t^{-} u\right|^{r}\right) d x\right] \\
& =\left(t^{-}\right)^{q+1} s^{\prime}\left(t^{-}\right)<0,
\end{aligned}
$$

and

$$
\begin{aligned}
\left\langle I_{\lambda}^{\prime}\left(t^{-} u\right), t^{-} u\right\rangle & =\left(t^{-}\right)^{p}\|u\|^{p}-\left(t^{-}\right)^{q} \lambda \int_{\Omega}\left(f|u|^{q}\right) d x-\left(t^{-}\right)^{r} \int_{\Omega}\left(h|u|^{r}\right) d x \\
& =\left(t^{-}\right)^{q}\left[s\left(t^{-}\right)-\lambda \int_{\Omega}\left(f|u|^{q}\right) d x\right]=0 .
\end{aligned}
$$

Thus $t^{-} u=t^{-}(u) u \in N_{\lambda}^{-}$, since for $t>t_{\max }$, we have

$$
\left\langle\psi^{\prime}(t u), t u\right\rangle=(p-q)\|t u\|^{p}-(r-q) \int_{\Omega}\left(h|t u|^{r}\right) d x<0, \quad \frac{d^{2}}{d t^{2}} I_{\lambda}(t u)<0,
$$

and

$$
\begin{aligned}
\frac{d}{d t} I_{\lambda}(t u) & =t^{p-1} \int_{\Omega}\left(a|\nabla u(x)|^{p}\right) d x-t^{q-1} \lambda \int_{\Omega}\left(f|u|^{q}\right) d x-t^{r-1} \int_{\Omega}\left(h|u|^{r}\right) d x \\
& =t^{p-1}\|u\|^{p}-t^{q-1} \lambda \int_{\Omega}\left(f|u|^{q}\right) d x-t^{r-1} \int_{\Omega}\left(h|u|^{r}\right) d x=0
\end{aligned}
$$

for $t=t^{-}$. Therefore $I_{\lambda}\left(t^{-} u\right)=\sup _{t \geq t_{\max }} I_{\lambda}(t u)$.

Case (II) If $\lambda \int_{\Omega}\left(f|u|^{q}\right) d x>0$, by (2.10) and

$$
s(0)=0<\lambda \int_{\Omega}\left(f|u|^{q}\right) d x \leq \lambda\|f\|_{L^{H}} S_{r}^{q}\|u\|^{q}<s\left(t_{\max }\right),
$$


for $\lambda \in\left(0, \lambda_{2}\right)$, there are unique $t^{+}$and $t^{-}$such that $0<t^{+}<t_{\max }<t^{-}$,

$$
s\left(t^{+}\right)=\lambda \int_{\Omega}\left(f|u|^{q}\right) d x=s\left(t^{-}\right),
$$

and $s^{\prime}\left(t^{+}\right)>0>s^{\prime}\left(t^{-}\right)$we have $t^{+}(u)(u) \in N_{\lambda}^{+}, t^{-}(u)(u) \in N_{\lambda}^{-}$and

$$
I_{\lambda}\left(t^{-}(u) u\right) \geq I_{\lambda}(t(u) u) \geq I_{\lambda}\left(t^{+}(u) u\right)
$$

for each $t(u) \in\left[t^{+}(u), t^{-}(u)\right]$ and $I_{\lambda}\left(t^{+}(u) u\right) \leq I_{\lambda}(t(u) u)$ for each $t(u) \in\left[0, t^{+}(u)\right]$, thus $I_{\lambda}\left(t^{-} u\right)=\sup _{t \geq t_{\max }} I_{\lambda}(t u), I_{\lambda}\left(t^{+} u\right)=\inf _{0 \leq t \leq t_{\max }} I_{\lambda}(t u)$.

(ii) by Case (II) of part (i).

(iii) Fix $u \in U$, define $G_{u}:(0, \infty) \times U \rightarrow \mathbf{R}$ by

$$
G_{u}(t, w)=\left\langle I_{\lambda}^{\prime}(t w), t w\right\rangle .
$$

Since $G_{u}\left(t^{-}(u), u\right)=\left\langle I_{\lambda}^{\prime}\left(t^{-}(u) u\right), t^{-}(u) u\right\rangle=0$, and

$$
\frac{\partial G_{u}}{\partial t^{-}}\left(t^{-}(u), u\right)=\left[t^{-}(u)\right]^{-1}\left\langle\psi_{\lambda}^{\prime}\left(t^{-}(u) u\right), t^{-}(u) u\right\rangle<0,
$$

then by the implicit function theorem, there is a neighborhood $W_{u}$ of $u$ in $U$ and an unique continuous function $T_{u}: W_{u} \rightarrow(0, \infty)$ such that $G_{u}\left(T_{u}(w), w\right)=0$ for all $w \in W_{u}$, in particular, $T_{u}(u)=t^{-}(u)$. Since $u \in U$ is arbitrary, we find that the function $T: U \rightarrow$ $(0, \infty)$, given by $T_{u}(u)=t^{-}(u)$ is continuous and one-to-one. Having $T^{-}: U \rightarrow N_{\lambda}^{-}$, where $T^{-}(u)=t^{-}(u) u$, we find that $T^{-}$is continuous and one-to-one. Now if $u \in N_{\lambda}^{-}$then we have $T^{-}(w)=u$, where $w=\frac{u}{\|u\|}$, since $t^{-}$is continuous on $U$, it follows that $t^{-}$is continuous for nonzero $u$. Then the proof is complete.

Lemma 2.8 There exists a positive number $\Lambda_{0} \leq \lambda_{1}\left(\lambda_{1}\right.$ defined in Lemma 2.4) such that if $\lambda \in\left(0, \Lambda_{0}\right)$, then

(i) $\alpha_{\lambda}^{+}<0$,

(ii) $\alpha_{\lambda}^{-}>0$ and $\alpha_{\lambda}^{+}<\alpha_{\lambda}^{-}$, in particular, $\alpha_{\lambda}=\alpha_{\lambda}^{+}$.

Proof (i) Let $u \in N_{\lambda}^{+}$, by (2.3)

$$
\left(\frac{r-p}{r-q}\right)\|u\|^{p}<\lambda \int_{\Omega}\left(f|u|^{q}\right) d x,
$$

and so

$$
\begin{aligned}
I_{\lambda}(u) & =\frac{r-p}{p r}\|u\|^{p}-\left(\frac{r-q}{q r}\right) \lambda \int_{\Omega}\left(f|u|^{q}\right) d x \\
& <-\frac{(r-p)(p-q)}{p q r}\|u\|^{p}<0 .
\end{aligned}
$$

Thus, $\alpha_{\lambda}^{+}<0$.

(ii) Let $u \in N_{\lambda}^{-}$, by (2.2) and the Sobolev embedding theorem,

$$
\left(\frac{p-q}{r-q}\right)\|u\|^{p}<\int_{\Omega}\left(h|u|^{r}\right) d x \leq S_{r}^{r}\|h\|_{L^{\infty}}\|u\|^{r},
$$


and so

$$
\|u\|>\left(\frac{p-q}{(r-q) S_{r}^{r}\|h\|_{L^{\infty}}}\right)^{\frac{1}{r-p}}
$$

for all $u \in N_{\lambda}^{-}$, by the proof of Lemma 2.3

$$
\begin{aligned}
I_{\lambda}(u) & \geq\|u\|^{q}\left[\left(\frac{r-p}{p r}\right)\|u\|^{p-q}-\left(\frac{r-q}{q r}\right) \lambda\|f\|_{L^{H}}\right] \\
& >\left(\frac{p-q}{(r-q) S_{r}^{r}\|h\|_{L^{\infty}}}\right)^{\frac{q}{r-p}}\left[\left(\frac{r-p}{p r}\right)\left(\frac{p-q}{(r-q) S_{r}^{r}\|h\|_{L^{\infty}}}\right)^{\frac{p-q}{r-p}}-\left(\frac{r-q}{q r}\right) \lambda\|f\|_{L^{H}}\right] .
\end{aligned}
$$

Thus, there exists a positive number $\Lambda_{0} \leq \lambda_{1}$ such that if $\lambda \in\left(0, \Lambda_{0}\right)$, then $I_{\lambda}(u)>0$, i.e., $\alpha_{\lambda}^{-}>0$ for all $u \in N_{\lambda}^{-}$. Obviously, $\alpha_{\lambda}^{+}<\alpha_{\lambda}^{-}$. This completes the proof.

\section{Proof of Theorem 2.1}

First, by following the idea of Tarantello [22], we have the following result.

Lemma 3.1 For each $u \in N_{\lambda}$, there exist $\epsilon>0$ and a differentiable function $\xi: B(0, \epsilon) \subset$ $X \rightarrow \mathbf{R}^{+}$such that $\xi(0)=1$, the function $\xi(v)(u-v) \in N_{\lambda}$ and

$$
\begin{aligned}
& \left\langle\xi^{\prime}(u), v\right\rangle \\
& =\frac{-p \int_{\Omega}\left(a|\nabla u|^{p-1} \nabla v+u^{p-1} v\right) d x+q \lambda \int_{\Omega}\left(f|u|^{q-2} u v\right) d x+r \int_{\Omega}\left(h|u|^{r-2} u v\right) d x}{(p-q)\|u\|^{p}-(r-q) \int_{\Omega}\left(h|u|^{r}\right) d x} \\
& \quad \text { for all } v(x) \in X .
\end{aligned}
$$

Lemma 3.2 For each $u \in N_{\lambda}^{-}$, there exist $\epsilon>0$ and a differentiable function $\xi^{-}: B(0, \epsilon) \subset$ $X \rightarrow \mathbf{R}^{+}$such that $\xi^{-}(0)=1$, the function $\xi^{-}(v)(u-v) \in N_{\lambda}^{-}$and

$$
\begin{aligned}
& \left\langle\xi^{-}(u), v\right\rangle \\
& =\frac{-p \int_{\Omega}\left(a|\nabla u|^{p-1} \nabla v+u^{p-1} v\right) d x+q \lambda \int_{\Omega}\left(f|u|^{q-2} u v\right) d x+r \int_{\Omega}\left(h|u|^{r-2} u v\right) d x}{(p-q)\|u\|^{p}-(r-q) \int_{\Omega}\left(h|u|^{r}\right) d x} \\
& \quad \text { for all } v(x) \in X .
\end{aligned}
$$

The proof of the two lemmas above is almost the same as given by Hsu [23] and thus we omit it.

Proposition 3.3 Let $\lambda_{0}=\min \left\{\lambda_{1}, \lambda_{2}, \Lambda_{0}\right\}$. Then for $\lambda \in\left(0, \lambda_{0}\right)$,

(i) there exists a minimizing sequence $\left\{u_{n}\right\} \subset N_{\lambda}$ such that

$$
\begin{aligned}
& I_{\lambda}\left(u_{n}\right)=\alpha_{\lambda}+o(1)=\alpha_{\lambda}^{+}+o(1), \\
& I_{\lambda}^{\prime}\left(u_{n}\right)=o(1) \quad \text { in } X^{-1} ;
\end{aligned}
$$


(ii) there exists a minimizing sequence $\left\{u_{n}\right\} \subset N_{\lambda}^{-}$such that

$$
\begin{aligned}
& I_{\lambda}\left(u_{n}\right)=\alpha_{\lambda}^{-}+o(1), \\
& I_{\lambda}^{\prime}\left(u_{n}\right)=o(1) \quad \text { in } X^{-1} .
\end{aligned}
$$

Proof (i) by Lemma 2.3, and the Ekeland variational principle [24], there exists a minimizing sequence $\left\{u_{n}\right\} \subset N_{\lambda}^{+}$such that

$$
\begin{aligned}
& I_{\lambda}\left(u_{n}\right)<\alpha_{\lambda}^{+}+\frac{1}{n}, \\
& I_{\lambda}\left(u_{n}\right)<I_{\lambda}(w)+\frac{1}{n}\left\|w-u_{n}\right\| \quad \forall w \in N_{\lambda}^{+} .
\end{aligned}
$$

By taking $n$ large, from Lemma 2.8(i), we have

$$
\begin{aligned}
I_{\lambda}\left(u_{n}\right) & =\left(\frac{1}{p}-\frac{1}{r}\right)\left\|u_{n}\right\|^{p}-\left(\frac{1}{q}-\frac{1}{r}\right) \lambda \int_{\Omega}\left(f\left|u_{n}\right|^{q}\right) d x \\
& <\alpha_{\lambda}^{+}+\frac{1}{n}<\frac{\alpha_{\lambda}^{+}}{2},
\end{aligned}
$$

consequently $u_{n} \neq 0$, and putting together (3.5), (2.6), and (2.7), we obtain for all $n$

$$
\left[-\frac{\alpha_{\lambda}^{+}}{2 \lambda\|f\|_{L^{h}} S_{r}^{q}}\left(\frac{q r}{r-q}\right)\right]^{\frac{1}{q}} \leq\left\|u_{n}\right\| \leq\left[\frac{p}{q} S_{r}^{q}\left(\frac{r-q}{r-p}\right)\left(\lambda\|f\|_{L^{H}}\right)\right]^{\frac{1}{p-q}} .
$$

Now, we will show that $\left\|I_{\lambda}^{\prime}\left(u_{n}\right)\right\| \rightarrow 0$, as $n \rightarrow \infty$.

Applying Lemma 3.1 with $u_{n}$ to obtain the functions $\xi^{+}: B(0, \epsilon) \rightarrow \mathbf{R}^{+}$for some $\epsilon_{n}>0$ such that $\xi^{+}(w)\left(u_{n}-w\right) \in N_{\lambda}^{+}$, choose $0<\rho<\epsilon_{n}$, let $u \in X$ with $u \neq \equiv 0$ and let $w_{\rho}=\frac{\rho u}{\|u\|}$. We set $\eta_{\rho}^{+}=\xi_{n}^{+}\left(w_{\rho}\right)\left(u_{n}-w_{\rho}\right)$, since $\eta_{\rho}^{+} \in N_{\lambda}^{+}$, we deduce from (3.4) that

$$
I_{\lambda}\left(\eta_{\rho}^{+}\right)-I_{\lambda}\left(u_{n}\right) \geq-\frac{1}{n}\left\|\eta_{\rho}^{+}-u_{n}\right\|,
$$

and by the mean value theorem, we have

$$
\left\langle I_{\lambda}^{\prime}\left(u_{n}\right), \eta_{\rho}^{+}-u_{n}\right\rangle+o\left(\left\|\eta_{\rho}^{+}-u_{n}\right\|\right) \geq-\frac{1}{n}\left\|\eta_{\rho}^{+}-u_{n}\right\| .
$$

Thus

$$
\begin{aligned}
& \left\langle I_{\lambda}^{\prime}\left(u_{n}\right),-w_{\rho}\right\rangle+\left(\xi_{n}^{+}\left(w_{\rho}\right)-1\right)\left\langle I_{\lambda}^{\prime}\left(u_{n}\right),\left(u_{n}-w_{\rho}\right)\right\rangle \\
& \geq-\frac{1}{n}\left\|\eta_{\rho}^{+}-u_{n}\right\|+o\left(\left\|\eta_{\rho}^{+}-u_{n}\right\|\right) .
\end{aligned}
$$

From $\xi_{n}^{+}\left(w_{\rho}\right)\left(u_{n}-w_{\rho}\right) \in N_{\lambda}^{+}$and (3.7) it follows that

$$
\begin{aligned}
& -\rho\left\langle I_{\lambda}^{\prime}\left(u_{n}\right), \frac{u}{\|u\|}\right\rangle+\left(\xi_{n}^{+}\left(w_{\rho}\right)-1\right)\left\langle I_{\lambda}^{\prime}\left(u_{n}\right)-I_{\lambda}^{\prime}\left(\eta_{\rho}^{+}\right),\left(u_{n}-w_{\rho}\right)\right\rangle \\
& \geq-\frac{1}{n}\left\|\eta_{\rho}^{+}-u_{n}\right\|+o\left(\left\|\eta_{\rho}^{+}-u_{n}\right\|\right) .
\end{aligned}
$$


Thus

$$
\begin{aligned}
\left\langle I_{\lambda}^{\prime}\left(u_{n}\right), \frac{u}{\|u\|}\right\rangle \leq & \frac{\left\|\eta_{\rho}^{+}-u_{n}\right\|}{n \rho}+\frac{o\left(\left\|\eta_{\rho}^{+}-u_{n}\right\|\right)}{\rho} \\
& +\frac{\left(\xi_{n}^{+}\left(w_{\rho}\right)-1\right)}{\rho}\left\langle I_{\lambda}^{\prime}\left(u_{n}\right)-I_{\lambda}^{\prime}\left(\eta_{\rho}^{+}\right),\left(u_{n}-w_{\rho}\right)\right\rangle .
\end{aligned}
$$

Since $\left\|\eta_{\rho}^{+}-u_{n}\right\| \leq \rho\left|\xi_{n}^{+}\left(w_{\rho}\right)\right|+\left|\xi_{n}^{+}\left(w_{\rho}-1\right)\right|\left\|u_{n}\right\|$ and $\lim _{\rho \rightarrow 0} \frac{\left|\xi_{n}^{+}\left(w_{\rho}\right)-1\right|}{\rho} \leq\left\|\xi_{n}^{\prime}(w)\right\|$, if we let $\rho \rightarrow 0$ in (3.8) for a fixed $n$, then by (3.6) we can find a constant $c>0$, independent of $\rho$, such that

$$
\left\langle I_{\lambda}^{\prime}\left(u_{n}\right), \frac{u}{\|u\|}\right\rangle \leq \frac{c}{n}\left(1+\left\|\xi_{n}^{\prime}(u)\right\|\right)
$$

and we are done once we show that $\left\|\left(\xi_{n}^{+}\right)^{\prime}(u)\right\|$ is uniformly bounded in $n$. By (3.1), (3.6), and (2.6), we have

$$
\left\langle\left(\xi_{n}^{+}\right)^{\prime}\left(u_{n}\right), v\right\rangle \leq \frac{b\|v\|}{\left|(p-q)\left\|u_{n}\right\|^{p}-(r-q) \int_{\Omega}\left(h\left|u_{n}\right|^{r}\right) d x\right|},
$$

for some $b>0$. We only need to show that

$$
\left|(p-q)\left\|u_{n}\right\|^{p}-(r-q) \int_{\Omega}\left(h\left|u_{n}\right|^{r}\right) d x\right|>c,
$$

for some $c>0$, and $n$ large enough. We argue by contradiction; assume that there exists a subsequence $\left\{u_{n}\right\}$ such that

$$
(p-q)\left\|u_{n}\right\|^{p}-(r-q) \int_{\Omega}\left(h\left|u_{n}\right|^{r}\right) d x=o(1)
$$

combining (3.10) with (3.6), we can find a suitable constant $d>0$ such that

$$
\int_{\Omega}\left(h\left|u_{n}\right|^{r}\right) d x \geq d
$$

for $n$ sufficiently large. In addition (3.10), and the fact that $u_{n} \in N_{\lambda}^{+}$also give

$$
\lambda \int_{\Omega}\left(f\left|u_{n}\right|^{q}\right) d x=\left\|u_{n}\right\|^{p}-\int_{\Omega}\left(h\left|u_{n}\right|^{r}\right) d x=\frac{r-p}{p-q} \int_{\Omega}\left(h\left|u_{n}\right|^{r}\right) d x+o(1),
$$

and the right side of (3.6) holds. This implies

$$
\begin{aligned}
I_{\lambda}(u) & =K(q, r)\left(\frac{\|u\|^{r}}{\int_{\Omega}\left(h|u|^{r}\right) d x}\right)^{\frac{p}{r-p}}-\lambda \int_{\Omega}\left(f|u|^{q}\right) d x \\
& =\frac{r-p}{p-q} \int_{\Omega}\left(h|u|^{r}\right) d x-\frac{r-p}{p-q} \int_{\Omega}\left(h|u|^{r}\right) d x=o(1) .
\end{aligned}
$$


However, by the right of (3.6), (3.11), and $\lambda \in\left(0, \lambda_{0}\right)$,

$$
\begin{aligned}
I_{\lambda}(u) & \geq K(q, r)\left(\frac{\|u\|^{r}}{\int_{\Omega}\left(h|u|^{r}\right) d x}\right)^{\frac{p}{r-p}}-\lambda\|f\|_{L^{H}} S_{r}^{r}\|u\|^{r} \\
& \geq K(q, r)(\tilde{c})^{\frac{p}{r-p}}-\lambda\|f\|_{L^{H}} S_{r}^{r}\|u\|^{r}>0,
\end{aligned}
$$

for $\lambda$ sufficiently small, here $\tilde{c}=\left(\|h\|_{L^{\infty}} S_{r}^{r}\right)^{-1}$. This contradicts (3.12), we get

$$
\left\langle I_{\lambda}^{\prime}\left(u_{n}\right), \frac{u}{\|u\|}\right\rangle \leq \frac{c}{n}
$$

this completes the proof of (i).

(ii) Similarly, by using Lemma 3.2, we can prove (ii) and thus its proof and its details are omitted here.

Theorem 3.4 Let $\lambda_{0}\left(q, p,\|h\|_{\infty}, r,\|f\|_{L^{H}}^{-1}\right)=\lambda_{0}=\min \left\{\lambda_{1}, \lambda_{2}, \Lambda_{0}\right\}$. Then for $\lambda \in\left(0, \lambda_{0}\right)$, the functional $I_{\lambda}$ has a minimizer point $u_{0}^{+}$in $N_{\lambda}^{+}$and it satisfies

(i) $I_{\lambda}\left(u_{0}^{+}\right)=\alpha_{\lambda}=\alpha_{\lambda}^{+}$,

(ii) $u_{0}^{+}$is a positive solution of equation $\left(E_{\lambda f, h}\right)$,

(iii) $\left\|u_{0}^{+}\right\| \rightarrow 0$ as $\lambda \rightarrow 0$.

Proof By Proposition 3.3(i), it follows that there exists $\left\{u_{n}\right\} \subset N_{\lambda}$ satisfying

$$
\begin{aligned}
& I_{\lambda}\left(u_{n}\right)=\alpha_{\lambda}+o(1)=\alpha_{\lambda}^{+}+o(1), \\
& I_{\lambda}^{\prime}\left(u_{n}\right)=o(1) \quad \text { in } X^{-1}(\Omega) .
\end{aligned}
$$

We can infer that $\left\{u_{n}\right\}$ is bounded from below on $X$. Thus, passing a subsequence if necessary, there exists $u_{0} \in X$, such that $u_{n} \rightarrow u_{0}$ weakly in $X$. We get $I_{\lambda}^{\prime}\left(u_{0}\right)=0$. Suppose, by absurdity, that $u_{0}=0$, then by $(2.2)$, we have $(p-q)\left\|u_{n}\right\|^{p}-(r-q) \int\left(h\left|u_{n}\right|^{r}\right) d x=o(1)$ and by (2.1), we get

$$
\begin{aligned}
\lambda \int_{\Omega}\left(f\left|u_{n}\right|^{q}\right) d x & =\left\|u_{n}\right\|^{p}-\int_{\Omega}\left(h\left|u_{n}\right|^{r}\right) d x+o(1)=\left\|u_{n}\right\|^{p}-\frac{p-q}{r-q}\left\|u_{n}\right\|^{p} \\
& =\frac{r-p}{r-q}\left\|u_{n}\right\|^{p}+o(1),
\end{aligned}
$$

thus by the Egorov theorem we obtain $\int_{\Omega}\left(f\left|u_{n}\right|^{q}\right) d x=o(1)$. Since $I_{\lambda}^{\prime}\left(u_{n}\right)=o(1)$ in $X^{-1}(\Omega)$, we have

$$
o(1)=\left\langle I_{\lambda}^{\prime}\left(u_{n}\right), u_{n}\right\rangle=\left\|u_{n}\right\|^{p}-\int_{\Omega}\left(h\left|u_{n}\right|^{r}\right) d x+o(1) .
$$

By Lemma 2.8(i), it follows that $\alpha_{\lambda}^{+}<0$, then considering $n_{0} \in \mathbf{N}$ such that

$$
\begin{aligned}
& \frac{1}{r}\left|\left\|u_{n}\right\|^{p}-\int_{\Omega}\left(h\left|u_{n}\right|^{r}\right) d x\right|+\frac{1}{q}\left|\lambda \int_{\Omega}\left(f\left|u_{n}\right|^{q}\right) d x\right|<-\frac{\alpha_{\lambda}^{+}}{4}, \\
& I_{\lambda}\left(u_{n}\right)<\frac{\alpha_{\lambda}^{+}}{2}
\end{aligned}
$$


for all $n \geq n_{0}$. Thus for all $n \geq n_{0}$, we get

$$
\left(\frac{1}{p}-\frac{1}{r}\right)\left\|u_{n}\right\|^{p}<I_{\lambda}\left(u_{n}\right)-\frac{\alpha_{\lambda}^{+}}{2}<0
$$

which is an absurdity, because $p<r$. Hence, $u_{0} \neq 0$ and since $I_{\lambda}^{\prime}\left(u_{0}\right)=0$, it follows that $u_{0} \in N_{\lambda}$ and in particular, $I_{\lambda}\left(u_{0}\right) \geq \alpha_{\lambda}$. We will show that, up to a subsequence, $u_{n} \rightarrow u_{0}$ strongly in $X$. Suppose, for a contradiction, that $\left\|u_{0}\right\|<\liminf _{n \rightarrow \infty}\left\|u_{n}\right\|$. Since $\left\{u_{n}\right\} \subset N_{\lambda}$ and $u_{0} \in N_{\lambda}$, we have

$$
\alpha_{\lambda}^{+} \leq I_{\lambda}\left(u_{0}\right)<\liminf _{n \rightarrow \infty} I_{\lambda}\left(u_{n}\right)=\alpha_{\lambda}^{+},
$$

which is a contradiction. Hence, we can suppose, up to a subsequence, that $u_{n} \rightarrow u_{0}$ strongly in $X$. Note that $u_{0} \in N_{\lambda}^{+}$, since $u_{0} \in N_{\lambda}$ and $I_{\lambda}\left(u_{0}\right)=\alpha_{\lambda}^{+}<\alpha_{\lambda}^{-}$. Considering $u_{0}^{+}=$ $\left|u_{0}\right|$, we get $u_{0}^{+} \not \equiv 0$ since $u_{0} \in X \backslash\{0\}$. If $u \in N_{\lambda}^{+}$, then $|u| \in N_{\lambda}^{+}$and by $I_{\lambda}\left(u_{0}^{+}\right)=I_{\lambda}\left(u_{0}\right)=\alpha_{\lambda}$ we see that $u_{0}^{+} \in N_{\lambda}$ is a local minimum point of $I_{\lambda}$ on $N_{\lambda}$. Then by Lemma 2.6. We find that $u_{0}^{+}$is a solution of the problem $\left(E_{\lambda f, h}\right)$. By the Harnack inequality according to Trudinger [25] we obtain $u_{0}^{+}>0$ in $\Omega$. Now by (2.3) we have

$$
\begin{aligned}
0 & <\left\langle\psi_{\lambda}^{\prime}\left(u_{0}^{+}\right), u_{0}^{+}\right\rangle=(p-r)\left\|u_{0}^{+}\right\|^{p}-(q-r) \lambda \int_{\Omega}\left(f\left|u_{0}^{+}\right|^{q}\right) d x \\
& \leq-(r-p)\left\|u_{0}^{+}\right\|^{p}+(r-q) \lambda\|f\|_{L^{H}}\left\|u_{0}^{+}\right\|^{q} .
\end{aligned}
$$

Then by (2.6), we infer that

$$
(r-p)\left\|u_{0}^{+}\right\|^{p} \leq(r-q) \lambda\|f\|_{L^{H}}\left\|u_{0}^{+}\right\|^{q}
$$

i.e.

$$
\left\|u_{0}^{+}\right\|^{p-q} \leq \frac{r-q}{r-p} \lambda\|f\|_{L^{H}}
$$

that is

$$
\left\|u_{0}^{+}\right\| \leq\left(\frac{r-q}{r-p}\right)^{\frac{1}{p-q}}\left(\lambda\|f\|_{L^{H}}\right)^{\frac{1}{p-q}}=c\left(\lambda\|f\|_{L^{H}}\right)^{\frac{1}{p-q}}
$$

where $c$ is a positive constant, independent of $\lambda$. So

$$
\left\|u_{0}^{+}\right\| \leq c\left(\lambda\|f\|_{L^{H}}\right)^{\frac{1}{p-q}}
$$

and thus we conclude the proof.

Next, we establish the existence of a local minimum for $I_{\lambda}$ on $N_{\lambda}^{-}$.

Theorem 3.5 Let $\lambda_{0}\left(q, p,\|h\|_{\infty}, r,\|f\|_{L^{H}}^{-1}\right)=\lambda_{0}=\min \left\{\lambda_{1}, \lambda_{2}, \Lambda_{0}\right\}$. Then for $\lambda \in\left(0, \lambda_{0}\right)$, the functional $I_{\lambda}$ has a minimizer point $u_{0}^{-}$in $N_{\lambda}^{-}$and it satisfies:

(i) $I_{\lambda}\left(u_{0}^{-}\right)=\alpha_{\lambda}^{-}$,

(ii) $u_{0}^{-}$is a positive solution of equation $\left(E_{\lambda f, h}\right)$. 
Proof By Proposition 3.3(ii), it follows that there exists a minimizing sequence $\left\{u_{n}\right\}$ for $I_{\lambda}$ on $N_{\lambda}^{-}$such that

$$
\begin{aligned}
& I_{\lambda}\left(u_{n}\right)=\alpha_{\lambda}^{-}+o(1), \\
& I_{\lambda}^{\prime}\left(u_{n}\right)=o(1) \quad \text { in } X^{-1}(\Omega) .
\end{aligned}
$$

By Lemma 2.8 and Lemma 2.3 and the compact embedding theorem, there exists a subsequence $\left\{u_{n}\right\}$ and $u_{0}^{-} \in N_{\lambda}^{-}$is a nonzero solution of $\left(E_{\lambda f, h}\right)$ such that

$$
\begin{aligned}
& u_{n} \rightarrow u_{0}^{-} \text {weakly in } X, \\
& u_{n} \rightarrow u_{0}^{-} \text {strongly in } L^{q}(\Omega) \text { and } L^{r}(\Omega) .
\end{aligned}
$$

We now prove that $u_{n} \rightarrow u_{0}^{-}$strongly in $X$. Suppose otherwise, then

$$
\left\|u_{0}^{-}\right\|<\liminf _{n \rightarrow \infty}\left\|u_{n}\right\|
$$

and so

$$
\begin{aligned}
& \left\|u_{0}^{-}\right\|^{p}-\lambda \int_{\Omega}\left(f\left|u_{0}^{-}\right|^{q}\right) d x-\int_{\Omega}\left(h\left|u_{0}^{-}\right|^{r}\right) d x \\
& \quad<\liminf _{n \rightarrow \infty}\left(\left\|u_{n}\right\|^{p}-\lambda \int_{\Omega}\left(f\left|u_{n}\right|^{q}\right) d x-\int_{\Omega}\left(h\left|u_{n}\right|^{r}\right) d x\right)=0 .
\end{aligned}
$$

This contradicts $u_{0}^{-} \in N_{\lambda}^{-}$. Hence, $u_{n} \rightarrow u_{0}^{-}$strongly in $X$. This implies $I_{\lambda}\left(u_{n}\right) \rightarrow I_{\lambda}\left(u_{0}^{-}\right)=$ $\alpha_{\lambda}^{-}$, as $n \rightarrow \infty$. Since $I_{\lambda}\left(u_{0}^{-}\right)=I_{\lambda}\left(\left|u_{0}^{-}\right|\right)$and $\left|u_{0}^{-}\right| \in N_{\lambda}^{-}$, by Lemma 2.6 , we may assume that $u_{0}^{-}$is a nonnegative solution. By Drábek, Kufner and Nicolosi [1, Lemma 2.1], we have $u_{0}^{-} \in L^{\infty}(\Omega)$. Then we can apply the Harnack inequality due to Trudinger [25] in order to find that $u_{0}^{-}$is positive in $\Omega$.

Now we can complete the proof of Theorem 2.1: By Theorem 3.4 and Theorem 3.5, for $\left(E_{\lambda f, h}\right)$ there exist two positive solutions $u_{0}^{+}$and $u_{0}^{-}$such that $u_{0}^{+} \in N_{\lambda}^{+}, u_{0}^{-} \in N_{\lambda}^{-}$. Since $N_{\lambda}^{+} \cap N_{\lambda}^{-}=\emptyset$, this implies that $u_{0}^{+}$and $u_{0}^{-}$are different. Thus the proof of Theorem 2.1 is complete.

\section{Time-fractional equations}

In this section, we switch our view point to the fractional order equation $\left(E_{\lambda f, h, t}\right)$ in weighted Sobolev space $H_{0}^{1}(a(x), \Omega)$ with the standard norm

$$
\|u\|_{H_{0}^{1}(a(x), \Omega)}=\left\{\int_{\Omega}\left(|a(x) \nabla u(x)|^{2}+(u(x))^{2}\right) d x\right\}^{\frac{1}{2}} .
$$

In order to discuss the existence of the positive solution for the $\left(E_{\lambda f, h, t}\right)$, we need to present some basic notations, definitions, and preliminary results, which will be used throughout this section. We first have the following two definitions by [18].

Definition 4.1 The Caputo fractional derivative of order $\alpha$ of a function $f(t), t>0$, is defined as

$$
D^{\alpha} f(t)=\frac{1}{\Gamma(1-\{\alpha\})} \int_{0}^{t} \frac{1}{(t-s)^{\{\alpha\}}} f^{([\alpha]+1)} d s,
$$


where $\{\alpha\},[\alpha]$ denotes the fractional and the integer part of the real number $\alpha$, respectively, and $\Gamma(\cdot)$ is the Gamma function.

Definition 4.2 The Riemann-Liouville fractional integral of order $\alpha$ of a function $f(t)$, $t>0$, is defined as

$$
I_{0^{+}}^{\alpha} f(t)=\frac{1}{\Gamma(\alpha)} \int_{0}^{t}(t-s)^{\alpha-1} f(s) d s
$$

provided that the right side is pointwise defined on $(0, \infty)$.

Lemma 4.1 [18] Assume $y \in C[0, T], T>0,1<\alpha<2$, then the problem

$$
D^{\alpha} u(t)=y(t), \quad t \in[0, T]
$$

has the unique solution

$$
u(t)=u(0)+u^{\prime}(0) t+\frac{1}{\Gamma(\alpha)} \int_{0}^{t}(t-s)^{\alpha-1} y(s) d s .
$$

Now we establish some results as regards the existence of positive solutions for $\left(E_{\lambda f, h, t}\right)$. By Lemma 4.1, we may reduce $\left(E_{\lambda f, h, t}\right)$ to an equivalent integral equation as in the following problem:

$$
\left(E _ { \lambda f , h , \text { integral } ) } \left\{\begin{array}{l}
-\phi(x)-\psi t+u(x, t) \\
=\frac{1}{\Gamma(\alpha)} \int_{0}^{t}(t-s)^{\alpha-1}\left(\operatorname{div}\left(a(x)|\nabla u(x, s)|^{p-2} \nabla u(x, s)\right)+(u(x, s))^{p-1}\right. \\
\left.\quad+\lambda f(x)|u(x, s)|^{q-1}+h(x)|u(x, s)|^{r-1}\right) d s, \quad \text { in } \Omega_{T}, \\
u(x, t)=0, \quad \text { on } \partial \Omega_{T} .
\end{array}\right.\right.
$$

The functional integral equations describe many physical phenomena in various areas of natural science, mathematical physics, mechanics, and population dynamics [26-29]. The theory of integral equations is developing rapidly with the help of tools in functional analysis, topology, and fixed point theory (see, for instance, [30-33]) and it serves as a useful tool in turn for other branches of mathematics, for example for differential equations (see [34-36]). Now we define

$$
\left(E_{\lambda f, h, \mathrm{fixed}}\right)\left\{\begin{aligned}
\Phi(u)= & \phi(x)+\psi(x) t \\
& +\frac{1}{\Gamma(\alpha)} \int_{0}^{t}(t-s)^{\alpha-1}\left(\operatorname{div}\left(a(x)|\nabla u(x, s)|^{p-2} \nabla u(x, s)\right)\right. \\
& +|u(x, s)|^{p-2} u(x, s)+\lambda f(x)|u(x, s)|^{q-1} \\
& \left.+h(x)|u(x, s)|^{r-1}\right) d s, \quad \text { in } \Omega_{T}, \\
u(x, t)= & 0, \quad \text { on } \partial \Omega_{T} .
\end{aligned}\right.
$$

Definition 4.3 We call $u \in C\left([0, T] ; H_{0}^{1}(a(x), \Omega)\right)$ a weak solution of the fractional order equation $\left(E_{\lambda f, h, t}\right)$, if $\int_{\Omega}(u-\Phi(u)) v d x=0, \forall t \in[0, T]$ for every $v \in H_{0}^{1}(a(x)$, $\Omega)$, i.e.

$$
\begin{aligned}
\int_{\Omega} u v d x= & \int_{\Omega}\left[\phi(x)+\psi(x) t+\frac{1}{\Gamma(\alpha)} \int_{0}^{t}(t-s)^{\alpha-1}\left(|u(x, s)|^{p-2} u(x, s)+\lambda f(x)|u(x, s)|^{q-1}\right.\right. \\
& \left.\left.+h(x)|u(x, s)|^{r-1}\right) d s\right] v d x \\
& -\int_{\Omega} \int_{0}^{t}(t-s)^{\alpha-1}\left(a(x)|\nabla u(x, s)|^{p-2} \nabla u(x, s)\right) d s v d x .
\end{aligned}
$$


Lemma 4.2 The operator $\Phi(u): H_{0}^{1}(a(x), \Omega) \rightarrow H^{-1}(a(x), \Omega)$ is completely continuous.

Proof Put

$$
\begin{aligned}
F(u)= & \operatorname{div}\left(a(x)|\nabla u(x, s)|^{p-2} \nabla u(x, s)\right) \\
& +(u(x, s))^{p-1}+\lambda f(x)|u(x, s)|^{q-1}+h(x)|u(x, s)|^{r-1} .
\end{aligned}
$$

We can rewrite

$$
\Phi(u)=\phi(x)+\psi(x) t+\frac{1}{\Gamma(\alpha)} \int_{0}^{t}(t-s)^{\alpha-1} F(u) d s .
$$

For each $v \in H_{0}^{1}(a(x), \Omega)$, and $\|v\|_{H_{0}^{1}(a(x), \Omega)}=1$, by integration by parts, we can get

$$
\left|\left\langle F(u), v||=\left.\left|\int\left(a(x)|\nabla u|^{p-1} \nabla v\right)+u^{p-1} v+\lambda f(x)\right| u\right|^{q-1} v+h(x)|u|^{r-1} v\right) d x\right| .
$$

Since $0 \leq f(x) \in L^{H}(\Omega)$, where $L^{H}(\Omega)=L^{\frac{r}{r-q}}(\Omega), q<r<p_{s}^{*}$, and $f(x)$ has a compact support in $\Omega, 0 \leq h(x) \in L^{\infty}(\Omega)$, and satisfies $h(x) \rightarrow 1$ as $|x| \rightarrow \infty$, so $F(u) \in C\left([0, T] ; H_{0}^{1}(a(x), \Omega)\right)$. Since $2<p$, by Sobolev embedding theorem, we have $W_{0}^{1, p}(a(x), \Omega) \hookrightarrow H_{0}^{1}(a(x), \Omega)$, and thus, $\|u\|_{H_{0}^{1}(a(x), \Omega)} \leq C\|u\|_{W_{0}^{1, p}(a(x), \Omega)}$, in the following, we denote $\|u\|_{H_{0}^{1}(a(x), \Omega)}$ and $\|u\|_{H^{-1}(a(x), \Omega)}$ by $\|u\|_{H_{0}^{1}},\|u\|_{H^{-1}}$, respectively. Hence, by the Cauchy-Schwarz inequalities, the Poincaré inequalities, the Hölder inequalities, the Sobolev embedding theorem, and (2.7) and $1<q<p<r$, we have

$$
\begin{aligned}
|\langle F(u), v\rangle| & \left.\left.\left|\int\left(a(x)|\nabla u|^{p-1} \nabla v\right)+u^{p-1} v+\lambda f(x)\right| u\right|^{q-1} v+h(x)|u|^{r-1} v\right) d x \mid \\
\leq & \left(\left.\left.\int|a(x)| \nabla u\right|^{p-1}\right|^{2} d x\right)^{\frac{1}{2}}\left(\int|\nabla v|^{2} d x\right)^{\frac{1}{2}}+\left(\int\left|u^{p-1}\right|^{2} d x\right)^{\frac{1}{2}}\left(\int|v|^{2} d x\right)^{\frac{1}{2}} \\
& +\left(\left.\left.\int|\lambda f(x)| u\right|^{q-1}\right|^{2} d x\right)^{\frac{1}{2}}\left(\int|v|^{2} d x\right)^{\frac{1}{2}}+\left(\left.\left.\int|h(x)| u\right|^{r-1}\right|^{2} d x\right)^{\frac{1}{2}}\left(\int|v|^{2} d x\right)^{\frac{1}{2}} \\
\leq & \left(\left.\left.\int|a(x)| \nabla u\right|^{p-1}\right|^{2} d x\right)^{\frac{1}{2}}\|\nabla v\|_{L^{2}(a(x), \Omega)}+\left(\int\left|u^{p-1}\right|^{2} d x\right)^{\frac{1}{2}}\|\nabla v\|_{L^{2}(a(x), \Omega)} \\
& +|\lambda|\|f(x)\|\left\|_{L^{H}}\left(\left.\left.\int|| u\right|^{q-1}\right|^{2} d x\right)^{\frac{1}{2}}\right\| \nabla v\left\|_{L^{2}(a(x), \Omega)}+\left(\left.\left.\int|| u\right|^{r-1}\right|^{2} d x\right)^{\frac{1}{2}}\right\| \nabla v \|_{L^{2}(a(x), \Omega)} \\
\leq & \left(\left(\left.\left.\int|a(x)| \nabla u\right|^{p-1}\right|^{2} d x\right)^{\frac{1}{2}}+\left(\int\left|u^{p-1}\right|^{2} d x\right)^{\frac{1}{2}}\right. \\
& \left.+|\lambda|\|f(x)\|_{L^{H}}\left(\left.\left.\int|| u\right|^{q-1}\right|^{2} d x\right)^{\frac{1}{2}}+\left(\left.\left.\int|| u\right|^{r-1}\right|^{2} d x\right)^{\frac{1}{2}}\right)\|v\|_{H_{0}^{1}} \\
\leq & \left(\left.\left.\int|a(x)| \nabla u\right|^{p-1}\right|^{2} d x\right)^{\frac{1}{2}}+\left(\int\left|u^{p-1}\right|^{2} d x\right)^{\frac{1}{2}} \\
& +|\lambda|\|f(x)\|{ }_{L^{H}}\left(\left.\left.\int|| u\right|^{q-1}\right|^{2} d x\right)^{\frac{1}{2}}+\left(\left.\left.\int|| u\right|^{r-1}\right|^{2} d x\right)^{\frac{1}{2}}
\end{aligned}
$$




$$
\begin{aligned}
\leq & \left(\int|a(x) \nabla u|^{2(p-1)} d x\right)^{\frac{1}{2(p-1)}(p-1)}+\left(\int|u|^{2(p-1)} d x\right)^{\frac{1}{2(p-1)}(p-1)} \\
& +|\lambda|\|f(x)\|_{L^{H}}\left(\int|u|^{2(q-1)} d x\right)^{\frac{1}{2(q-1)}(q-1)}+\left(\int|u|^{2(r-1)} d x\right)^{\frac{1}{2(r-1)}(r-1)} \\
= & \|a(x) \nabla u\|_{L^{2(p-1)}(a(x), \Omega)}^{(p-1)}+\|u\|_{L^{2(p-1)}(a(x), \Omega)}^{(p-1)} \\
& +|\lambda|\|f(x)\|_{L^{H}}\|u\|_{L^{2(q-1)}(a(x), \Omega)}^{(q-1)}+\|u\|_{L^{2(r-1)}(a(x), \Omega)}^{(r-1)} \\
\leq & C_{0}\|u\|_{H_{0}^{1}}^{(p-1)}+C_{1}|\lambda|\|f(x)\|_{L^{H}}\|\nabla u\|_{L^{2}(a(x), \Omega)}^{(q-1)}+C_{2}\|\nabla u\|_{L^{2}(a(x), \Omega)}^{(r-1)} \\
\leq & C\|u\|_{X}^{(p-1)}+C_{3}\left(|\lambda|\|f(x)\|_{L^{H}}\|u\|_{H_{0}^{1}}^{(q-1)}+\|u\|_{H_{0}^{1}}^{(r-1)}\right) \\
\leq & C\|u\|_{X}^{(p-1)}+C_{4}\left(|\lambda|\|f(x)\|_{L^{H}}\|u\|_{X}^{(q-1)}+\|u\|_{X}^{(r-1)}\right) \\
\leq & \widetilde{C}\left\{\left(\frac{r-q}{r-p}\right)^{\frac{1}{p-q}} S_{r}^{\frac{q}{p-q}}\left(\lambda\|f\|_{\left.L^{H}\right)^{\frac{1}{p-q}}}^{(p-1)}\right\}^{(p-p)}\right. \\
& +|\lambda|\|f(x)\|_{L^{H}}\left\{\left(\frac{r-q}{r-p}\right)^{\frac{1}{p-q}} S_{r}^{\frac{q}{p-q}}\left(\lambda\|f\|_{L^{H}}\right)^{\frac{1}{p-q}}\right\}^{(q-1)} \\
& +\left\{\left(\frac{r-q}{r-p}\right)^{\frac{1}{p-q}} S_{r}^{\frac{q}{p-q}}\left(\lambda\|f\|_{\left.L^{H}\right)^{\frac{1}{p-q}}}^{(r-1)}\right\}^{(r-p)}\right. \\
& M .
\end{aligned}
$$

Here, $C_{0}, C_{1}, C_{2}, C_{3}, C_{4}, C$ denote the best Sobolev constants, and $\widetilde{C}=\max \left\{C, C_{4}\right\}$.

Thus, by Cauchy-Schwarz inequalities, we obtain

$$
\begin{aligned}
\|\Phi(u)\|_{H^{-1}} & =\sup _{\|v\|_{H_{0}^{1}} \leq 1}|\langle\Phi(u), v\rangle| \\
= & \sup _{\|v\|_{H_{0}^{1}} \leq 1}\left|\langle\phi(x), v\rangle+\langle\psi(x), v\rangle t+\frac{1}{\Gamma(\alpha)} \int_{0}^{t}(t-s)^{\alpha-1}\langle F(u), v\rangle d s\right| \\
\leq & |\langle\phi(x), v\rangle|+|\langle\psi(x), v\rangle t|+\left|\frac{1}{\Gamma(\alpha)} \int_{0}^{t}(t-s)^{\alpha-1}\langle F(u), v\rangle d s\right| \\
\leq & \|\phi(x)\|_{L^{\infty}(a(x), \Omega)}\|v\|_{H_{0}^{1}}+\|\psi(x)\|_{L^{\infty}(a(x), \Omega)}\|v\|_{H_{0}^{1}} T \\
& +|\langle F(u), v\rangle| \frac{1}{\Gamma(\alpha)} \int_{0}^{t}(t-s)^{\alpha-1} d s \mid \\
\leq & \|\phi(x)\|_{L^{\infty}(a(x), \Omega)}+\|\psi(x)\|_{L^{\infty}(a(x), \Omega)} T+\frac{M}{\Gamma(\alpha)}\left|\int_{0}^{t}(t-s)^{\alpha-1} d s\right| \\
\leq & \|\phi(x)\|_{L^{\infty}(a(x), \Omega)}+\|\psi(x)\|_{L^{\infty}(a(x), \Omega)} T+\frac{M}{\alpha \Gamma(\alpha)} t^{\alpha} \\
\leq & \|\phi(x)\|_{L^{\infty}(a(x), \Omega)}+\|\psi(x)\|_{L^{\infty}(a(x), \Omega)} T+\frac{M}{\alpha \Gamma(\alpha)} T^{\alpha} .
\end{aligned}
$$

Hence, $\Phi(u)$ is bounded. 
On the other hand, given $\epsilon>0$, setting

$$
\delta=\left(\|\psi(x)\|_{L^{\infty}(a(x), \Omega)}+\frac{M}{\Gamma(\alpha)} T^{\alpha-1}\right)^{-1} \epsilon,
$$

then, for every $v \in H_{0}^{1}(a(x), \Omega), t_{1}<t_{2}, t_{1}, t_{2} \in[0, T]$, and $t_{2}-t_{1}<\delta$, one has $\| \Phi u\left(t_{2}\right)-$ $\Phi u\left(t_{1}\right) \|_{H^{-1}}=\sup _{\|v\|_{H_{0}^{1}} \leq 1}\left|\left\langle\Phi u\left(t_{2}\right)-\Phi u\left(t_{1}\right), v\right\rangle\right| \leq \epsilon$. That is to say, $\Phi(u)$ has equicontinuity. In fact,

$$
\begin{aligned}
\| \Phi & u\left(t_{2}\right)-\Phi u\left(t_{1}\right) \|_{H^{-1}} \\
= & \sup _{\|v\|_{H_{0}^{1}} \leq 1}\left|\left\langle\Phi u\left(t_{2}\right)-\Phi u\left(t_{1}\right), v\right\rangle\right| \\
= & \sup _{\|v\|_{H_{0}^{1}} \leq 1} \mid\langle\psi(x), v\rangle\left(t_{2}-t_{1}\right)+\frac{1}{\Gamma(\alpha)} \int_{0}^{t_{2}}\left(t_{2}-s\right)^{\alpha-1}\langle F(u), v\rangle d s \\
& -\frac{1}{\Gamma(\alpha)} \int_{0}^{t_{1}}\left(t_{1}-s\right)^{\alpha-1}\langle F(u), v\rangle d s \mid \\
\leq & \|\psi(x)\|_{L^{\infty}(a(x), \Omega)}\|v\|_{H_{0}^{1}}\left|t_{2}-t_{1}\right|+|\langle F(u), v\rangle|\left|\frac{1}{\Gamma(\alpha)} \int_{t_{1}}^{t_{2}}\left(t_{2}-s\right)^{\alpha-1} d s\right| \\
& +\frac{1}{\Gamma(\alpha)} \int_{0}^{t_{1}}|\langle F(u), v\rangle|\left|\left(t_{2}-s\right)^{\alpha-1}-\left(t_{1}-s\right)^{\alpha-1}\right| d s \\
\leq & \|\psi(x)\|_{L^{\infty}(a(x), \Omega)}\left|t_{2}-t_{1}\right|+\frac{M}{\alpha \Gamma(\alpha)} t_{2}^{\alpha}-\frac{M}{\alpha \Gamma(\alpha)} t_{1}^{\alpha} \\
\leq & \|\psi(x)\|_{L^{\infty}(a(x), \Omega)}\left|t_{2}-t_{1}\right|+\frac{M}{\alpha \Gamma(\alpha)}\left(t_{2}^{\alpha}-t_{1}^{\alpha}\right) .
\end{aligned}
$$

In the following, we divide the proof into two cases.

Case 1: $\delta \leq t_{1}<t_{2}<T$, since $1<\alpha<2$, we get

$$
\begin{aligned}
& \left\|\Phi u\left(t_{2}\right)-\Phi u\left(t_{1}\right)\right\|_{H^{-1}} \\
& =\sup _{\|v\|_{H_{0}^{1}} \leq 1}\left|\left\langle\Phi u\left(t_{2}\right)-\Phi u\left(t_{1}\right), v\right\rangle\right| \\
& \leq\|\psi(x)\|_{L^{\infty}(a(x), \Omega)}\left|t_{2}-t_{1}\right|+\frac{M}{\alpha \Gamma(\alpha)}\left(t_{2}^{\alpha}-t_{1}^{\alpha}\right) \\
& =\|\psi(x)\|_{L^{\infty}(a(x), \Omega)}\left|t_{2}-t_{1}\right|+\frac{M}{\alpha \Gamma(\alpha)} \alpha t^{\alpha-1}\left(t_{2}-t_{1}\right) \\
& =\|\psi(x)\|_{L^{\infty}(a(x), \Omega)} \delta+\frac{M}{\Gamma(\alpha)} T^{\alpha-1} \delta \\
& \leq\|\psi(x)\|_{L^{\infty}(a(x), \Omega)} \delta+\frac{M}{\Gamma(\alpha)} T^{\alpha-1} \delta \\
& =\left(\|\psi(x)\|_{L^{\infty}(a(x), \Omega)}+\frac{M}{\Gamma(\alpha)} T^{\alpha-1}\right) \delta \leq \epsilon .
\end{aligned}
$$

Here, $t_{1}<t<t_{2}$, and we apply the mean theorem $t_{2}^{\beta}-t_{1}^{\beta}=\beta t^{\beta-1}\left(t_{2}-t_{1}\right)$. 
Case 2: $0 \leq t_{1}, t_{2}<\alpha^{\frac{1}{\alpha}} \delta$

$$
\begin{aligned}
\left\|\Phi u\left(t_{2}\right)-\Phi u\left(t_{1}\right)\right\|_{H^{-1}} & =\sup _{\|v\|_{H_{0}^{1}} \leq 1}\left|\left\langle\Phi u\left(t_{2}\right)-\Phi u\left(t_{1}\right), v\right\rangle\right| \\
& \leq\|\psi(x)\|_{L^{\infty}(a(x), \Omega)}\left|t_{2}-t_{1}\right|+\frac{M}{\alpha \Gamma(\alpha)}\left(t_{2}^{\alpha}-t_{1}^{\alpha}\right) \\
& \leq\|\psi(x)\|_{L^{\infty}(a(x), \Omega)} \delta+\frac{M}{\alpha \Gamma(\alpha)}\left(\alpha^{\frac{1}{\alpha}} \delta\right)^{\alpha} \\
& \leq\|\psi(x)\|_{L^{\infty}(a(x), \Omega)} \delta+\frac{M}{\Gamma(\alpha)} T^{\alpha-1} \delta \\
& =\left(\|\psi(x)\|_{L^{\infty}(a(x), \Omega)}+\frac{M}{\Gamma(\alpha)} T^{\alpha-1}\right) \delta \leq \epsilon .
\end{aligned}
$$

By applying the Arzela-Ascoli theorem, we know that $\Phi(u): H_{0}^{1}(a(x), \Omega) \rightarrow H^{-1}(a(x), \Omega)$ is completely continuous. This completes the proof.

By Lemma 4.2, we know that $\int_{\Omega}(u-\Phi(u)) v d x=0, \forall t \in[0, T]$ for every $v \in H_{0}^{1}(a(x), \Omega)$. That is to say, the fractional order equation $\left(E_{\lambda f, h, t}\right)$ has a unique weak solution $u \in$ $C\left([0, T] ; H_{0}^{1}(a(x), \Omega)\right)$.

\section{Conclusion}

In this paper, we study the existence of positive solutions for $P$-Laplace semi-linear elliptic equations and the corresponding time-fractional equations. That is, we first establish the multiplicity of positive solutions for nonlinear elliptic equations with a positive smooth weight function involving concave and convex nonlinearities in weighted Sobolev spaces, and the proof of the two positive solutions for the problem $\left(E_{\lambda f, h}\right)$ is given. Second, by applying the Arzela-Ascoli fixed point theorem, one existence result for the time-fractional equations is also obtained.

Finally we like to mention that for $\left(E_{\lambda f, h}\right)$ its corresponding time-fractional equations are the foundation models of the nonlinear problems in the field of PDES and it is worthwhile to pay more attention to their study.

\section{Competing interests}

The authors declare that they have no competing interests.

Authors' contributions

All authors contributed equally to the writing of this paper. All authors read and approved the final manuscript.

\section{Author details}

'School of Mathematics and Statistics, Xi'an Jiaotong University, Xi'an, 710049, China. ${ }^{2}$ Department of Mathematics, Yunnan Nationalities University, Kunming, 650031, China. ${ }^{3}$ Institute of Mathematics, Yunnan Normal University, Kunming, 650092, China. ${ }^{4}$ Department of Mathematics, Tongji University, Shanghai, 200092, China.

\section{Acknowledgements}

The third author is grateful to Professor Boling Guo and Professor Zhouping Xin for their support. The project is supported by the National Natural Science Foundation of China (Nos. 11161057, 10971164).

Received: 20 March 2014 Accepted: 13 June 2014 Published: 22 Jul 2014

\section{References}

1. Drábek, P, Kufner, A, Nicolosi, F: Quasilinear Elliptic Equations with Degenerations and Singularities. de Gruyter, Berlin (1997)

2. Adams, RA, John, JFF: Sobolev Space. Academic Press, New York (2009) 
3. Ambrosetti, A, Brezis, H, Cerami, G: Combined effects of concave and convex nonlinearities in some elliptic problems. J. Funct. Anal. 122, 519-543 (1994)

4. Adimurthi, FP, Yadava, L: On the number of positive solutions of some semilinear Dirichlet problems in a ball. Differ. Integral Equ. 10, 1157-1170 (1997)

5. Damascelli, L, Grossi, M, Pacella, F: Qualitative properties of positive solutions of semilinear elliptic equations in symmetric domains via the maximum principle. Ann. Inst. Henri Poincaré, Anal. Non Linéaire 16, 631-652 (1999)

6. Tang, M: Exact multiplicity for semilinear Dirichlet problem involving concave and convex nonlinearities. Proc. R. Soc. Edinb., Sect. A 133, 705-717 (2003)

7. Wu, TF: On semilinear elliptic equations involving concave-convex nonlinearities and sign-changing weight function. J. Math. Anal. Appl. 318, 253-270 (2006)

8. Miotto, ML, Miyagaki, OH: Multiple positive solutions for semilinear Dirichlet problems with sign-changing weight function in infinite strip domains. Nonlinear Anal. 71, 3434-3447 (2009)

9. Wu, TF: Multiple positive solutions for Dirichlet problems involving concave and convex nonlinearities. Nonlinear Anal. 69, 4301-4323 (2008)

10. Lakshmikantham, V, Leela, S, Vasundhara, DJ: Theory of Fractional Dynamic Systems. Cambridge Academic Publishers, Cambridge (2009)

11. Li, CF, Luo, XN, Zhou, Y: Existence of positive solutions of the boundary value problem for nonlinear fractional differential equations. Comput. Math. Appl. 59(3), 1363-1375 (2010)

12. Miller, KS, Ross, B: An Introduction to the Fractional Calculus and Fractional Differential Equations. Wiley, New York (1993)

13. Podlubny, I: Fractional Differential Equations. Mathematics in Science and Engineering. Academic Press, New York (1999)

14. Samko, SG, Kilbas, AA, Marichev, OI: Fractional Integrals and Derivatives, Theory and Applications. Gordon \& Breach, Yverdon (1993)

15. Tian, Y, Chen, A: The existence of positive solution to three-point singular boundary value problem of fractional differential equation. Abstr. Appl. Anal. (2009). doi:10.1155/2009/314656

16. Zhang, SQ: Positive solutions for boundary value problem of nonlinear fractional differential equations. Electron. J. Differ. Equ. 2006, 36 (2006)

17. Bai, Z, Lu, H: Positive solutions for boundary value problem of nonlinear fractional differential equations. J. Math. Anal. Appl. 311(2), 495-505 (2005)

18. Kilbas, AA, Srivastava, HM, Trujillo, J: Theory and Applications of Fractional Differential Equations. Elsevier, Amsterdam (2006)

19. Kilbas, AA, Trujillo, JJ: Differential equations of fractional order: methods, results and problems, II. Appl. Anal. 81, 435-493 (2002)

20. Rabinowitz, PH: Minimax Methods in Critical Point Theory with Applications to Differential Equations. Regional Conf. Ser. in Math. Am. Math. Soc., Providence (1986)

21. Brown, KJ, Zhang, YP: The Nehari manifold for a semilinear elliptic problem with a sign-changing weight function. J. Differ. Equ. 193, 481-499 (2003)

22. Tarantello, G: On nonhomogeneous elliptic involving critical Sobolev exponent. Ann. Inst. Henri Poincaré, Anal. Non Linéaire 9, 281-304 (1992)

23. Hsu, TS: Multiplicity results for $p$-Laplacian with critical nonlinearity of concave-convex type and sign-changing weight functions. Abstr. Appl. Anal. 2009, 652109 (2009)

24. Ekland, I: On the variational principle. J. Math. Anal. Appl. 17, 324-353 (1974)

25. Trudinger, NS: On Harnack type inequalities and their application to quasilinear elliptic equations. Commun. Pure Appl. Math. 20, 721-747 (1967)

26. Corduneanu, C: Integral Equations and Applications. Cambridge University Press, New York (1973)

27. O'Regan, D: Existence theory for nonlinear Volterra integrodifferential and integral equations. Nonlinear Anal. 31, 317-341 (1998)

28. Jeribi, A: A nonlinear problem arising in the theory of growing cell populations. Nonlinear Anal., Real World Appl. 3 , 85-105 (2002)

29. Ben Amar, A, Jeribi, A, Mnif, M: Some fixed point theorems and application to biological model. Numer. Funct. Anal. Optim. 29, 1-23 (2008)

30. Barroso, CS: Krasnoselskii's fixed point theorem for weakly continuous maps. Nonlinear Anal. 55, 25-31 (2003)

31. Ben Amar, A, Jeribi, A, Mnif, M: On a generalization of the Schauder and Krasnosel'skii fixed point theorems on Dunford-Pettis spaces and applications. Math. Methods Appl. Sci. 28, 1737-1756 (2005)

32. Latrach, K, Taoudi, MA, Zeghal, A: Some fixed point theorems of the Schauder and the Krasnosel'skii type and application to nonlinear transport equations. J. Differ. Equ. 221, 256-271 (2006)

33. Zhang, SS: Integral Equation. Chongqing (1988)

34. Zhang, SS, Yang, GS: Some further generalizations of Ky Fan's minimax inequality and its applications to variational inequalities. Appl. Math. Mech. 11(11), 1027-1034 (1990)

35. Zhang, SS, Yang, GS: On the solution by determinantal series to Volterra integral equation of the second kind with convolution-type kernel. Math. Numer. Sin. 10(2), 146-157 (1988)

36. Willem, M: Minimax Theorems. Birkhäuser, Boston (1996)

10.1186/1029-242X-2014-262

Cite this article as: Qiu et al.: Positive solutions for $P$-Laplace problems with nonlinear time-fractional differential equation. Journal of Inequalities and Applications 2014, 2014:262 